\title{
Clinical Differences in Types of Otalgia
}

\author{
Sang Hoon Kim, Tae Hyun Kim, Jae Yong Byun, Moon Suh Park, and Seung Geun Yeo \\ Department of Otorhinolaryngology-Head and Neck Surgery, Kyung Hee University School of Medicine, Seoul, Korea
}

\author{
Received October 7, 2014 \\ Revised December 12, 2014 \\ Accepted March 27, 2015
}

\author{
Address for correspondence \\ Seung Geun Yeo, MD, PhD \\ Department of Otorhinolaryngology- \\ Head and Neck Surgery, \\ Kyung Hee University \\ School of Medicine, \\ 23 Kyungheedae-ro, \\ Dongdaemun-gu, Seoul 130-872, \\ Korea \\ Tel +82-2-958-8474 \\ Fax +82-2-958-8470 \\ E-mail yeo2park@gmail.com
}

\begin{abstract}
Background and Objectives: Although otalgia is usually associated with ear problems, it may also originate outside the ear. We therefore assessed the clinical characteristics of patients with otalgia. Subjects and Methods: We analyzed 294 patients who presented with otalgia. We assessed differences in otalgia between adults and children, differences in otogenic vs. referred otalgia between adults and children, differences between men and women. $\mathbf{R e}-$ sults: Of the 294 patients, 208 (70.7\%) had otogenic otalgia and 86 (29.3\%) had referred otalgia. Hearing disturbance and otorrhea were significantly more common in otogenic otalgia, whereas rhinorrhea, sore throat, and postnasal drip were significantly more common in referred otalgia. Children were more likely to have otogenic otalgia than adults. The proportion of patients with referred otalgia was significantly higher in adults than in children $(p<0.05)$. Otogenic otalgia was more common in men, whereas referred otalgia was more common in women. Among patients with referred otalgia, neuralgia was significantly more frequent in women than in men $(p<0.05)$. Conclusions: Otogenic otalgia was more frequent in men than in women and in children than in adults, whereas referred otalgia was more frequent in women and adults, indicating that types of otalgia were dependent on age and gender.
\end{abstract}

J Audiol Otol 2015;19(1):34-38

KEY WORDS: Otalgia · Primary · Referred · Adult · Child.

\section{Introduction}

Various diseases can cause otalgia because the ear has rich sensory innervationthrough many cranial (V, VII, IX, and X) and cervical (2 and 3 ) nerves. Otalgia can be classified as primary or referred. Primary otalgia is ear pain that originates inside the ear, whereas referred otalgia is ear pain that originates from outside the ear [1,2]. Thus, a detailed patient history and through exam are is required to accurately determine whether otalgia is primary or referred.

Each part of the ear is affected by several sensory nerves. The auricle is affected by cranial nerves V, VII, X, C2, and C3; the external auditory meatus by cranial nerves V, VII, and X; the tympanic membrane by cranial nerves VII, IX, and X; and the middle ear by cranial nerves V, VII, and IX. These 4 cranial nerves, as well as cervical nerves 2 and 3 are also distributed in other regions of the body as well as the ears. Therefore phys-

This is an Open Access article distributed under the terms of the Creative Commons Attribution Non-Commercial License (http://creativecommons. org/licenses/by-nc/3.0/) which permits unrestricted non-commercial use, distribution, and reproduction in any medium, provided the original work is properly cited. ical exam in adjacent structures should be performed [3-5]. We therefore investigated the clinical characteristics of patients with otalgia, including accompanying diseases, the difference between children and adults, and the difference between men and women.

\section{Subjects and Methods}

Between 2010 and 2013, 294 patients visited the Department of Otolaryngology at Kyung Hee University Medical Center with complaints of otalgia. Patients were subdivided into those with otogenic or referred otalgia and their clinical characteristics were compared. In addition, we assessed differences in characteristics of otalgia between adults and children, differences in characteristics of otogenic vs. referred otalgia between adults and children, differences between men and women, and differences according to the side of pain. Patients who had difficulties communicating and those with an inaccurate medical history were excluded.

The 294 patients consisted of 108 males and 186 females. There were 52 children, of mean age $5.5 \pm 2.7$ years (range, 1 
to 13 years) and 231 adults, of mean age $49.8 \pm 15.8$ years (range, 20 to 81 years). The patients underwent thorough examinations covered the ear, the teeth, the temporomandibular joint, the nose, the sinuses, and head and neck areas, and when necessary, examinations included direct and indirect laryngoscopy and biopsy. Patients without an ear, nose, and throat disease were referred to other departments for a definitive diagnosis.

All statistical analyses were performed using SPSS version 18.0 (SPSS Inc., Chicago, IL, USA). Groups were compared using the chi-square test, Fisher's exact test, and other statistical analyses, as indicated. Differences were considered statistically significant at $p<0.05$.

\section{Results}

Of the 294 patients, 208 (70.7\%) were diagnosed with otogenic otalgia and $86(29.3 \%)$ with referred otalgia. Children were significantly more likely to have otogenic otalgia than adults $(p<0.05)$. Most children with otogenic otalgia were diagnosed with acute otitis media (AOM). The frequency of re- ferred otalgia was significantly higher in adults than in children. Otogenic otalgia tended to be more frequent in males than in females, whereas referred otalgia was more frequent in females $(p<0.05)$. Neuralgia was diagnosed significantly more often in females (Table 1).

Ear fullness (33\%) and rhinorrhea (14.6\%) were the most common symptoms accompanying otogenic and referred otalgia, respectively. Hearing disturbance and otorrhea were significantly more frequent in patients with otogenic otalgia, whereas rhinorrhea, sore throat, and postnasal drip were significantly more frequent in patients with referred otalgia $(p<0.05$ each; Table 2 and 3$)$. There was no different in frequency in unilateral and bilateral otalgia in otogenic and referred otalgia (Table 4).

\section{Discussion}

Otalgia can be classified into 2 types. Otogenic otalgia originates from diseases of the external, middle and inner ear, whereas referred otalgia arises from pathologies outside the ear $[4,5]$. The frequency and ratio of these two types of symp-

Table 1. Diagnosis of otalgia patients

\begin{tabular}{|c|c|c|c|c|c|c|c|}
\hline & Total (\%) & Children (\%) & Adults (\%) & $p$-value & Male (\%) & Female (\%) & p-value \\
\hline \multicolumn{8}{|l|}{ Otogenic otalgia (diagnosis) } \\
\hline COM & $73(35.1)$ & $11(21.2)$ & $58(25.1)$ & 0.549 & $33(30.6)$ & $40(21.5)$ & 0.083 \\
\hline $\mathrm{AOM}$ & $55(26.4)$ & $26(50.0)$ & $28(12.1)$ & $0.001 *$ & $25(23.1)$ & $30(16.1)$ & 0.137 \\
\hline External auditory canal & $33(15.9)$ & $5(9.6)$ & $27(11.7)$ & 0.670 & $13(12.0)$ & $20(10.8)$ & 0.737 \\
\hline Ear trauma & $13(6.2)$ & $1(1.9)$ & $10(4.3)$ & 0.417 & $7(6.5)$ & $6(3.2)$ & 0.191 \\
\hline E-tube dysfunction & $9(4.3)$ & & $9(3.9)$ & & $4(3.7)$ & $5(2.7)$ & 0.626 \\
\hline Ramsay hunt syndrome/Bell's palsy & $8(3.8)$ & & $8(3.5)$ & & $2(1.9)$ & $6(3.2)$ & 0.485 \\
\hline Auricular infection & $7(3.4)$ & & $7(3.0)$ & & $1(0.9)$ & $6(3.2)$ & 0.212 \\
\hline Myringitis & $2(1.0)$ & & $2(0.9)$ & & $0(0)$ & $2(1.1)$ & \\
\hline Impacted cerumen & $1(0.5)$ & & $1(0.4)$ & & $1(0.9)$ & $0(0)$ & \\
\hline Others & $7(3.4)$ & & $7(3.0)$ & & $3(2.8)$ & $4(2.2)$ & \\
\hline Total & & $43(82.7)$ & $157(68.0)$ & $0.035^{*}$ & $89(82.4)$ & $119(64.0)$ & $0.001 *$ \\
\hline \multicolumn{8}{|l|}{ Referred otalgia (diagnosis) } \\
\hline Tonsillitis, pharyngitis & $19(22.1)$ & $4(7.7)$ & $14(6.1)$ & 0.663 & $4(3.7)$ & $15(8.1)$ & 0.143 \\
\hline Nasal lesion & $17(20.0)$ & $3(5.8)$ & $14(6.1)$ & 0.936 & $5(4.6)$ & $12(6.5)$ & 0.519 \\
\hline Neuralgia (CN V, CN IX) & $12(14.0)$ & & $12(5.2)$ & & $0(0)$ & $12(6.5)$ & $0.007^{*}$ \\
\hline Headache & $10(11.6)$ & & $10(4.3)$ & & $1(0.9)$ & $9(4.8)$ & 0.074 \\
\hline TMJ disorder & $7(8.1)$ & & $7(3.0)$ & & $1(0.9)$ & $6(3.2)$ & 0.212 \\
\hline Oral lesion & $2(2.3)$ & & $2(0.9)$ & & $0(0)$ & $2(1.1)$ & \\
\hline LPR & $2(2.3)$ & & $2(0.9)$ & & $1(0.9)$ & $1(0.5)$ & \\
\hline Salivary gland disorder & $2(2.3)$ & $1(1.9)$ & $1(0.4)$ & & $1(0.9)$ & $1(0.5)$ & \\
\hline Cervical lymphadenophathy & $1(1.2)$ & & $1(0.4)$ & & $1(0.9)$ & $0(0)$ & \\
\hline Nasopharyngeal cancer & $1(1.2)$ & & $1(0.4)$ & & $1(0.9)$ & $0(0)$ & \\
\hline Others & $13(15.1)$ & $1(1.9)$ & $10(4.3)$ & & $4(3.7)$ & $9(4.8)$ & \\
\hline Total & & $9(17.3)$ & $74(32.0)$ & $0.039 *$ & $19(17.6)$ & $67(36.0)$ & $0.001^{*}$ \\
\hline
\end{tabular}

* $p<0.05$, Fisher's exact test. COM: chronic otitis media, AOM: acute otitis media, TMJ: temporomandibular joint, LPR: laryngopharyngeal reflux 
toms are dependent on whether patients are seen by otolaryngologists or other physicians. In this study, otogenic otalgia was more frequent than referred otalgia, perhaps because these patients were initially examined and treated by otologists.

Otogenic otalgia may be due to inflammation, mechanical causes, neoplasm, or Eustachian tube dysfunction [6]. Infectious causes include furunculosis, infected sebaceous cysts, cellulitis, otitis externa, necrotizing otitis externa, AOM, mastoiditis, petrous apicitis, bullous otitis externa, and bullous myringitis. Inflammatory causes include chondrodermatitis and relapsing polychondritis. Mechanical causes include trau-

Table 2. Symptoms accompanying otalgia

\begin{tabular}{lll}
\hline & \multicolumn{1}{c}{$\begin{array}{c}\text { Accompanying } \\
\text { symptoms }\end{array}$} & $\mathrm{n}(\%)$ \\
\hline Otologic symptoms & Ear fullness & $97(33.0)$ \\
& Hearing disturbance & $90(30.6)$ \\
& Otorrhea & $79(26.9)$ \\
& Tinnitus & $65(22.1)$ \\
& Dizziness & $38(12.9)$ \\
Non-otologic & Rhinorrhea & $43(14.6)$ \\
symptoms & Nasal obstruction & $40(13.6)$ \\
& Sore throat & $29(9.9)$ \\
& Fever & $23(7.8)$ \\
& Postnasal drip & $20(6.8)$ \\
& Voice change & $3(1.6)$ \\
& Reflux symptom & $3(1.6)$ \\
\hline
\end{tabular}

matic laceration, pinna hematoma, and tympanic membrane perforation. Neoplastic causes include squamous cell carcinoma and adenocarcinoma; and Eustachian tube dysfunctions include those due to otitis media with effusion and chronic otitis media.

Acute infectious ear disease is the most common cause of primary otalgia. Patients with chronic otologic infections, except for chronic myringitis, rarely complain of severe otalgia. Thus, another cause, such as acute infection, is likely if chronic otologic infections are accompanied by otalgia [7].

The causes of otalgia varied among our patients. For example, $78 \%$ of patients with primary otalgia were diagnosed with acute infectious ear disease, similar to previous results [4].

Referred otalgia may be caused by various mechanisms. Complex sensory innervation of the auricle and external ear originates during the generation of the ear. Otic cysts are located between pairs of branchial arches, with cyst location resulting in the domination of various cranial nerves. Other organs are also under the control of these sensory nerves, resulting in referred otalgia [8,9]. Referred otalgia may arise from the inability of the brain to distinguish the origin of pain because neurons starting from visceral and somatic sensation territories ascend to the brain via the same pathway [10]. Alternatively, miscommunication may occur at the ventral posterior nucleus between the lateral and medial levels of the thalamus. Finally, referred otalgia may be a high-level phenomenon oc-

Table 3. Main symptoms accompanying otogenic and referred otalgia

\begin{tabular}{lccc}
\hline Accompanying symptoms & Otogenic otalgia (\%) & Referred otalgia (\%) & p value \\
\hline Ear fullness & $73(35.1)$ & $24(27.9)$ & 0.233 \\
Hearing disturbance & $73(35.1)$ & $17(19.8)$ & $0.009^{*}$ \\
Otorrhea & $72(34.6)$ & $7(8.1)$ & $0.000^{*}$ \\
Tinnitus & $47(22.6)$ & $18(20.9)$ & 0.754 \\
Dizziness & $29(13.9)$ & $9(10.5)$ & 0.419 \\
Rhinorrhea & $25(12.0)$ & $18(20.9)$ & $0.049^{*}$ \\
Nasal obstruction & $25(12.0)$ & $15(17.4)$ & 0.217 \\
Sore throat & $14(6.7)$ & $15(17.4)$ & $0.005^{*}$ \\
Fever & $18(8.7)$ & $5(5.8)$ & 0.409 \\
Postnasal drip & $10(4.8)$ & $10(11.6)$ & $0.035^{*}$ \\
Voice change & $1(0.5)$ & $2(2.3)$ & 0.206 \\
Reflux symptom & $1(0.5)$ & $2(2.3)$ & 0.206 \\
\hline
\end{tabular}

*p<0.05, Fisher's exact test

Table 4. Difference in laterality between otogenic and referred otalgia

\begin{tabular}{lccc}
\hline & Otogenic otalgia & Referred otalgia & p value \\
\hline Unilateral & $174(83.7 \%)$ & $64(74.4 \%)$ & $32(37.2 \%)$ \\
Right & $96(46.2 \%)$ & $32(37.2 \%)$ & 0.155 \\
Left & $78(37.5 \%)$ & $22(25.6 \%)$ & \\
\hline
\end{tabular}

Chi-square test 
curring at the cerebral cortex itself [8].

Referred otalgia is associated with the nerve affected. For example, the auriculotemporal nerve (cranial nerve V) is affected by temporomandibular joint dysfunction, dental diseases, trigeminal neuralgia, and mandibular osteomyelitis/tumor. The posterior auricular nerve (cranial nerve VII) is affected by acoustic neuroma and herpes zoster infection. Jacobson's nerve (cranial nerve IX) is affected by tonsillitis/pharyngitis, sinusitis, pharyngeal tumor, and glossopharyngeal neuroma. Arnold's nerve (cranial nerve $\mathrm{X}$ ) is affected by laryngopharyngeal reflux, cricopharyngeal spasm, and vagal stimulators. The greater auricular (C2) and lesser occipital (C3) nerves are affected by cervical spine degenerative disease, cervical root cysts, ArnoldChiari type, whiplash, vascular diseases, fibromyalgia, and other psychogenic causes [11]. Therefore, a detailed medical history and a physical examination of all parts of the head and neck are required for patients with otalgia $[12,13]$.

The presence of lesions in organs other than the ear in patients who complain of otalgia may delay diagnosis and treatment. In this study, we took detailed medical histories, excluding patients for whom it was impossible to get a medical history or for whom medical records were incomplete. However, one patient $(0.4 \%)$ diagnosed with nasopharyngeal cancer was included. The only presenting symptom in this patient was otalgia and the physical examination at the first visit included only the ears, nose, and head \& neck. Two months later, this patient had progressed to a sore throat along with the symptoms of otitis media. Nasal endoscopy revealed a mass in the nasopharynx, with cancer diagnosed after performing $\mathrm{CT}$ and biopsy.

The causes of otalgia differed in children and adults. Diseases of the ear were the most common causes of otalgia in children, whereas secondary or referred otalgia was more common in adults $[14,15]$. The most common causes of primary and secondary otalgia in children are AOM, common colds and other upper respiratory tract infections, otitis externa, foreign bodies, recurrent parotitis, tonsillitis, quinsy, neck abscess, sinusitis/nasal causes, temporomandibular joint dysfunction, dental causes and rare causes [14]. In this study, AOM was the most frequent cause of otogenic otalgia $(50 \%)$, followed by chronic otitis media, otitis externa and ear trauma. Referred otalgia usually arose from a disease such as tonsillitis, pharyngitis or rhinosinusitis, accompanied by systemic symptoms. Children were diagnosed with otogenic otalgia significantly more frequently than adults because the incidence of AOM was higher in children than in adults. Referred otalgia was less common in children than in adults, perhaps because otogenic otalgia is more frequent in children than in adults. In this study otogenic otalgia was more common than referred otalgia in adults; however referred otalgia was more frequent in adults than in children.

This study found that otogenic otalgia was more frequent in men, whereas referred otalgia was more frequent in women. Males may be more likely than females to pick their ears, thus leading to infectious ear diseases and the more frequent occurrence of otogenic otalgia in males. Further studies will be needed to address this hypothesis.

The region, duration, and severity of pain, as well as its sudden or gradual onset, are useful in diagnosing otalgia. Also, it is essential to confirm the pattern of accompanying symptoms. In this study, otologic symptoms, such as ear fullness, hearing disturbance, otorrhea, tinnitus, and dizziness, accompanied otogenic otalgia; whereas rhinorrhea, nasal obstruction, postnasal drip, sore throat, fever, voice change, and reflux symptoms accompanied referred otalgia. This result is consistent with those of previous reports $[16,17]$.

In children, otalgia, cough, rhinitis, fever, diarrhea or vomiting are symptoms of AOM, the most commonly diagnosed cause of primary otalgia [18]. The rates of referred otalgia were similar in men and women. Previous studies found that unilateral otalgia was more common than bilateral otalgia, although the frequencies of right and left sided unilateral otalgia were similar [19]. We found that $83.7 \%$ of patients with otogenic otalgia and $74.4 \%$ of those with referred otalgia had unilateral otalgia, but there was no difference between the right and left sides.

\section{Conclusion}

Otogenic otalgia was more commonly diagnosed than referred otalgia, with the latter usually arising from rhinologic or head and neck diseases. The proportion of otogenic and referred otalgia differed by age and gender, with otogenic otalgia being more frequent in men and in children and referred otalgia more frequent in women and in adults. Adults who complain of otalgia should be suspected of having referred otalgia and undergo detailed physical examination for other diseases that can cause referred otalgia.

\section{Acknowledgments}

This work was supported by the National Research Foundation of Korea (NRF) grant funded by the Korea government (MSIP) (No. 2011-0030072).

\section{REFERENCES}

1) Majumdar S, Wu K, Bateman ND, Ray J. Diagnosis and management of otalgia in children. Arch Dis Child Educ Pract Ed 2009;94: 33-6.

2) Murtagh J. The painful ear. Aust Fam Physician 1991;20:1779-83. 
3) Wazen JJ. Referred otalgia. Otolaryngol Clin North Am 1989;22: 1205-15.

4) Shah RK, Blevins NH. Otalgia. Otolaryngol Clin North Am 2003;36: 1137-51.

5) Reiss M, Reiss G. [Differential diagnosis of otalgia]. Schmerz 1999; 13:392-7.

6) Visvanathan V, Kelly G. 12 minute consultation: an evidence-based management of referred otalgia. Clin Otolaryngol 2010;35:409-14

7) Osguthorpe JD, Nielsen DR. Otitis externa: review and clinical update. Am Fam Physician 2006;74:1510-6.

8) Scarbrough TJ, Day TA, Williams TE, Hardin JH, Aguero EG, Thomas CR Jr. Referred otalgia in head and neck cancer: a unifying schema. Am J Clin Oncol 2003;26:e157-62.

9) Charlett SD, Coatesworth AP. Referred otalgia: a structured approach to diagnosis and treatment. Int J Clin Pract 2007;61:1015-21.

10) Yanagisawa K, Kveton JF. Referred otalgia. Am J Otolaryngol 1992;13:323-7.

11) Jaber JJ, Leonetti JP, Lawrason AE, Feustel PJ. Cervical spine causes for referred otalgia. Otolaryngol Head Neck Surg 2008;138:479-85.

12) CKim DS, Cheang P, Dover S, Drake-Lee AB. Dental otalgia. J Laryngol Otol 2007;121:1129-34.

13) Ely JW, Hansen MR, Clark EC. Diagnosis of ear pain. Am Fam Physician 2008;77:621-8

14) Majumdar $\mathrm{S}, \mathrm{Wu} \mathrm{K}$, Bateman ND, Ray J. Diagnosis and management of otalgia in children. Arch Dis Child Educ Pract Ed 2009;94: 33-6.

15) Leung AK, Fong JH, Leong AG. Otalgia in children. J Natl Med Assoc 2000;92:254-60.

16) Chen RC, Khorsandi AS, Shatzkes DR, Holliday RA. The radiology of referred otalgia. AJNR Am J Neuroradiol 2009;30:1817-23.

17) Neilan RE, Roland PS. Otalgia. Med Clin North Am 2010;94:961-71.

18) Worrall G. Acute earache. Can Fam Physician 2011;57:1019-21, e320-2.

19) Taziki MH, Behnampour N. A study of the etiology of referred otalgia. Iran J Otorhinolaryngol 2012;24:171-6. 